\title{
Percepção dos enfermeiros sobre o significado dos cuidados paliativos em pacientes com câncer terminal
}

\author{
The perception by nurses of the significance of palliative care \\ in patients with terminal cancer
}

\author{
Maria Andréa Fernandes ${ }^{1}$ \\ Carla Braz Evangelista ${ }^{1}$ \\ Indiara Carvalho dos Santos Platel ${ }^{1}$ \\ Glenda Agra ${ }^{2}$ \\ Marineide de Souza Lopes ${ }^{2}$ \\ Francileide de Araújo Rodrigues ${ }^{1}$
}

\footnotetext{
${ }^{1}$ Núcleo de Estudos e Pesquisas em Bioética, Centro de Ciências da Saúde, Universidade Federal da Paraíba. Cidade Universitária - Campus I, Castelo Branco. 58.059-900 João Pessoa PB. m.andreaf@hotmail.com ${ }^{2}$ Universidade Federal de Campina Grande.
}

\begin{abstract}
This study sought to assess the perception of nurses with respect to cancer patients under palliative care. It is an exploratory study with a qualitative approach conducted with nurses from a hospital attending cancer patients under palliative care located in the city of Joano Pessoa, State of Paraiba. The study included nine nurses who worked in the hospital. The empirical material was collected using the technique of semi-structured interviews and analyzed using the content analysis technique. The interpretative analysis of the interviews led to the definition of three categories: Improving the quality of life through the alleviation of pain and suffering; Palliative Care: $a$ multi-professional study of terminal patients and their families in the grieving process; Communication: a source of dignity in the terminal care process. The conclusion reached is that the study revealed that the nurses involved acknowledge the importance of the multidisciplinary team. It enables the nurses to reflect on the use of communication as an essential element of care for patient and family under palliative care. It is hoped that the data obtained may foster further research on the topic.
\end{abstract}

Key words Palliative care, Nursing, Terminal care
Resumo Este estudo teve como objetivo conhecer a percepção do enfermeiro diante do paciente com câncer sob cuidados paliativos. Trata-se de um estudo exploratório com abordagem qualitativa, realizado com enfermeiros assistenciais de um hospital que atende pacientes oncológicos em regime de cuidados paliativos, situado no município de João Pessoa (PB). Participaram do estudo nove enfermeiros, que atuavam no referido hospital. O material empírico foi coletado utilizando-se a técnica de entrevista semiestruturada e analisado mediante a técnica de análise de conteúdo. A análise interpretativa das entrevistas possibilitou a construção de três categorias: Promoção da qualidade de vida através do alivio da dor e sofrimento; Cuidados Paliativos: um olhar multiprofissional para o paciente terminal e família no processo de luto; Comunicação: fonte de dignidade no processo de terminalidade. Concluise que o estudo possibilitou evidenciar que os enfermeiros envolvidos reconhecem a importância da equipe multiprofissional, propiciando ao enfermeiro reflexões acerca do uso da comunicação como elemento essencial do cuidar para paciente e família sob cuidados paliativos. Espera-se que os dados obtidos possam subsidiar novas investigações sobre a temática.

Palavras-chave Cuidados paliativos, Enfermagem, Assistência terminal 


\section{Introdução}

$\mathrm{O}$ ato de cuidar é uma atividade eminentemente humana que visa promover o bem-estar do ser fragilizado. O cuidado é parte integrante da vida; sem ele, o ser humano não conseguiria sobreviver. É uma relação de afetividade que se configura numa atitude de responsabilidade, atenção, preocupação e envolvimento com o cuidador e o ser cuidado ${ }^{1}$.

No tocante ao cuidar, especificamente com o paciente acometido por uma patologia em estágio avançado e sem perspectivas de cura, a atenção e o cuidado estão direcionados em suas necessidades e limitações, uma vez que o processo de morte é irreversível e o tempo de sobrevida está restrito há dias, semanas ou meses².

Com base nesse entendimento, torna-se essencial adotar uma prática assistencial que esteja fundamentada no bem-estar biopsicossocial e espiritual da pessoa em sua finitude, a fim de proporcionar uma melhor qualidade de vida e minimizar o sofrimento durante a doença termi$\mathrm{nal}^{3}$. Dessa maneira, devem-se considerar, essencialmente, os cuidados paliativos, como modalidade de assistência, pois, exige da equipe um olhar atento e cauteloso.

Baseada em uma visão holística do ser humano os cuidados paliativos têm como filosofia valorizar a vida e encarar a morte como um processo natural. Assim, não adia e nem prolonga a morte, mas ampara o ser em suas angústias e medos provendo o alívio da dor e de outros sintomas, oferecendo suporte para que os pacientes possam viver o mais ativamente possível, ajudando a família e os cuidadores no processo de luto ${ }^{4}$.

Portanto, é evidente a importância dos cuidados paliativos direcionados ao paciente na terminalidade da vida, especialmente o oncológico, visto que estes cuidados proporcionam uma abordagem diferenciada de tratamento que tem como objetivo principal a promoção do cuidar humanizado. Visto que dirige seu foco para o alívio das necessidades biopsicossociais e espirituais, assim como integra a esses cuidados valores, crenças, práticas culturais e religiosas do paciente e seus familiares ${ }^{5}$.

Cumpre assinalar, que nesta modalidade de cuidar, o enfermeiro torna-se capaz de ver o mundo e oferecer seus fundamentos e práticas essenciais para assistir, cuja prioridade é valer-se de habilidades profissionais para aliviar o sofrimento do paciente em todas as suas formas ${ }^{1,6}$. Contudo, para a obtenção desses propósitos é de suma importância que esse profissional pro- mova uma assistência pautada no respeito, na humanização e no acolhimento.

Com base nesse entendimento, torna-se essencial adotar uma prática assistencial que esteja fundamentada no bem-estar biopsicossocial e espiritual da pessoa em sua finitude, a fim de proporcionar uma melhor qualidade de vida e minimizar o sofrimento durante a doença terminal.3 Dessa maneira, devem-se considerar, essencialmente, os cuidados paliativos, como modalidade de assistência, pois exige da equipe um olhar atento e cauteloso. Considerando a relevância da temática, este estudo parte da seguinte questão norteadora: Qual a percepção de enfermeiro diante de paciente com câncer sob cuidados paliativos? Para responder este questionamento, o estudo tem como objetivo: conhecer a percepção de enfermeiro diante de paciente com câncer sob cuidados paliativos.

\section{Metodologia}

Para a realização da pesquisa, optou-se por um estudo exploratório, com abordagem qualitativa. A pesquisa foi realizada em um hospital que atende pacientes oncológicos em regime de cuidados paliativos no município de João Pessoa (PB). A população do estudo envolveu enfermeiros assistenciais que prestam cuidados direcionados ao paciente com câncer em fase terminal na referida instituição. Para a seleção da amostra foram considerados os seguintes critérios de inclusão: que o enfermeiro estivesse em atividade laboral durante o período de coleta de dados, apresentar, no mínimo, um ano de atuação profissional na instituição selecionada e que aceitasse participar do estudo. Desse modo, participaram da pesquisa nove enfermeiros assistenciais.

Para a coleta de dados procedeu-se uma entrevista semiestruturada contendo questões pertinentes ao objetivo do estudo realizado no período de setembro a dezembro de 2012. Os dados foram analisados por meio da técnica de Análise de Conteúdo ${ }^{7}$ que pressupõe as seguintes fases: pré-análise, exploração do material e tratamento dos resultados, inferência e interpretação. Após a organização do material, foram identificadas as unidades temáticas que emergiram dos depoimentos dos participantes do estudo. Essas unidades foram agrupadas por convergência dos significados, classificadas e agregadas em categorias que definiram a especificação dos temas e os mesmos foram analisados com subsídio da literatura com a finalidade de apreender as propostas da investigação. 
Vale observar que durante a coleta do material empírico, foi apresentado aos participantes o Termo de Consentimento Livre e Esclarecido, estabelecido pela Resolução 196/96, que determina as diretrizes e as normas regulamentadoras para pesquisas envolvendo seres humanos, esclarecendo o objetivo da pesquisa, a garantia do seu anonimato e o sigilo de dados confidenciais ${ }^{8}$. O projeto do qual decorre este estudo obteve aprovação do Comitê de Ética em Pesquisa, do Hospital Universitário Lauro Wanderley (CEP/HULW). É oportuno aludir que, para garantir o anonimato dos participantes do estudo, eles foram codificados, de forma genérica, de Enf 1 a Enf 9.

\section{Resultados e Discussão}

Os enfermeiros participantes do estudo ao exporem suas concepções acerca do que consideram cuidados paliativos, trazem as peculiaridades de um cuidado que vem sendo aperfeiçoado ao longo do tempo profissional na oncologia, principalmente no que se refere aos voltados para o alívio da dor e do sofrimento com a finalidade de promover qualidade de vida, no compartilhar da existência junto aos pacientes terminais e seus familiares.

Diante dos dados apresentados, deu-se seguimento à análise, na qual foi realizada uma leitura criteriosa das respostas. Buscando alcançar o objetivo proposto desta pesquisa, os textos foram agrupados conforme os núcleos de sentido que apresentavam, os quais foram aproximados à temática e deram origem a três categorias, cujo conteúdo desvela o olhar sensível do enfermeiro ao paciente e família com as abordagens de cuidado, a saber: "Promoção de qualidade de vida através do alívio da dor e sofrimento"; "Cuidados Paliativos: um olhar para o paciente e família no processo de terminalidade e luto"; "Comunicação: fonte de dignidade no processo de terminalidade".

Categoria I - Promoção da qualidade de vida através do alivio da dor e sofrimento

A primeira categoria expressa a percepção dos enfermeiros em relação ao paciente terminal oncológico sob cuidados paliativos. Observa-se a tendência dos participantes da pesquisa em relacionar a qualidade de vida do enfermo ao alívio da dor e do sofrimento demostrados a seguir:

Os Cuidados Paliativos são cuidados que o profissional proporciona ao paciente sem perspectiva de cura $[. .$.$] no caso do paciente com câncer terminal$ esse cuidado atende as necessidades do indivíduo para obter uma melhor qualidade de vida. (Enf 1)

É uma filosofia de cuidar que se aplica a pacientes portadores de patologias crônicas que não tenha perspectiva de cura a fim de estabelecer melhor qualidade de vida ao doente aliviando suas dores e sofrimento. (Enf 5)

São cuidados prestados que visam o conforto e bem estar do paciente, minimizando os sintomas da patologia terminal melhorando a qualidade de vida [...] (Enf 7)

Esse entendimento vai ao encontro da filosofia dos cuidados paliativos que propõe oferecer o conforto e alívio necessários para minimizar o sofrimento e a dor do paciente, ofertando portanto, qualidade de vida componente essencial para manter a dignidade na finitude humana.

O sofrimento é uma condição humana e afeta a pessoa em seus aspectos biopsicosocial e espiritual. Frequentemente esse sofrimento é potencializado quando a pessoa está vivenciando uma enfermidade que ameaça a vida, pois a dor é percebida como uma experiência que se caracteriza pela subjetividade e multidimensionalidade e pode manifestar-se por meio de sinais fisiológicos e/ou emocionais. Nesse sentido, o enfermeiro é o profissional da área da saúde que permanece mais tempo próximo ao paciente; assim, tem responsabilidades no manejo da dor, proporcionando alívio do sofrimento e melhora da qualidade de vida ${ }^{3}$.

Nesta perspectiva, ao avaliar corretamente a presença de dor nestes pacientes, o enfermeiro deve considerar os diferentes instrumentos disponíveis utilizados para caracterizar a dor, lembrando que a sua escolha depende da condição física, da idade e da condição de comunicação que o paciente apresenta. Nesse sentido, vale ressaltar que o conceito de 'dor total', introduzido por Saunders ${ }^{9}$, enfatiza a importância de interpretar o fenômeno doloroso não somente na sua dimensão física, mas também nos seus aspectos emocionais, sociais e espirituais que influenciam na gênese e na expressão da queixa dolorosa que a percepção de morte lhe traz.

Nessa conjuntura, o enfermeiro consegue prestar uma assistência que visa à qualidade de vida e à manutenção do conforto, atuando, nas mais diversas tecnologias no auxílio das funções fisiológicas. Evidencia-se nas falas abaixo que os Participantes da pesquisa compreendem que paliar, também, é proporcionar o conforto. Como mostram os trechos dos seguintes relatos:

Cuidado prestado para aliviar o sofrimento do paciente portador de uma patologia crônica e sem 
cura, proporcionando conforto e minimizando as complicações, sendo de grande importância para o paciente com câncer terminal e sua família [...]. Os Cuidados Paliativos é uma modalidade de cuidar que valoriza o ser, suas queixas e proporciona o conforto [...] (Enf 2)

É uma modalidade de cuidar que promove conforto, [...] qualidade de vida dos pacientes e seus familiares, em fase terminal atuando por meio do [...] alívio do sofrimento [...] (Enf 8)

Esses cuidados visam à promoção de conforto e são voltados para higiene, alimentação, curativos, e atenção sobre analgesia, observando-se, portanto, as necessidades de diminuição de sofrimento para manutenção da qualidade de vida. O sofrimento causado pela dor do paciente em cuidados paliativos não se restringe à dor física gerada pelo tumor, mas se constitui também como reflexo da condição vivenciada. O sofrimento vivido na fase terminal da doença é muito mais que físico, afeta o ser intrínseca e extrinsecamente, o todo que se conecta consigo, com os outros e com o mundo ${ }^{6,10}$.

Vale ressaltar que atualmente existem vários modalidades terapêuticas nos cuidados paliativos, no entanto cabe aos profissionais de saúde ampliar seus conhecimentos para que possam usá-los a favor da prevenção e do alívio do sofrimento, contribuindo desse modo, para um cuidado mais humanizado. Estudos ${ }^{11}$ corroboram evidenciando que o simples ato de ouvir música pode produzir mudanças positivas no humor, restaurar a paz e o equilíbrio emocional, proporcionar relaxamento e facilitar a expressão de sentimentos como: tristeza, raiva e luto. Também possibilita o estímulo à memória afetiva, podendo proporcionar um ambiente seguro para a retomada de sentimentos e lembranças do passado, para a sensação de alívio e de renovação, tão importantes na fase final de vida. A musicoterapia é capaz de aliviar a solidão e o desgosto e reduzir a dor e o impacto da doença ao proporcionar prazer e alegria.

É oportuno destacar que os participantes da pesquisa enfatizam a valorização da qualidade de vida e que para isso os pacientes terminais devem permanecer junto à família, recebendo tratamento adequado e conforto. $\mathrm{O}$ paciente não deve ser considerado apenas como um corpo doente, mas como uma pessoa que carrega consigo uma história de vida constituída de medos, anseios e desejos. Cabe à equipe, em especial ao enfermeiro atuar de forma ativa e efetiva, esclarecendo as dúvidas e encorajando atitudes positivas.
Categoria II - Cuidados Paliativos: um olhar multiprofissional para o paciente terminal e família no processo de luto

Os participantes da pesquisa relatam que os cuidados paliativos promovem uma assistência integral, humanizada e multidisciplinar e visa à minimização dos anseios do paciente e da família e dar suporte terapêutico durante o processo de enlutamento, como pode ser evidenciado nos depoimentos que se seguem:

[...] além de apoiar os familiares durante o luto [...] (Enf 4)

[...] esse cuidado que [...] ameniza os anseios e apoia a família principalmente no período do luto (Enf 9)

Sendo necessária uma equipe multidisciplinar para atuar nesses cuidados, mesmo durante o luto (Enf 6)

[...] a Equipe deve ser multidisciplinar e apoiar o paciente de forma a promover uma assistência humanizada e dar suporte à família durante o processo de terminalidade e de luto (Enf 2)

Diante desses depoimentos, evidencia-se que os cuidados paliativos promovem uma assistência humanizada que integra não somente o paciente como também a sua família na prestação de cuidados por parte dos profissionais de saúde. Os cuidados paliativos ofertam apoio à família durante o processo de luto, promovendo diminuição do medo e angústia que cerca o momento difícil pelo qual estão passando.

O luto é uma das áreas importantes de intervenção da equipe de cuidados paliativos, que contempla o empreendido pelo paciente e pela família desde o diagnóstico de uma doença sem possibilidades terapêuticas de cura até o processo de morte e morrer. É no processo de luto que o paciente tem a possibilidade de aprender que a morte deve ser tomada como real e é a partir desta experiência que o mesmo estabelece novas concepções sobre o mundo, possibilitando investimentos pessoais, ou seja, favorecendo o enfrentamento para o paciente viver seu próprio luto e reorganizar sua vida nesses momentos que lhe restam ${ }^{12,13}$.

Os cuidados paliativos promovem o alívio da dor e de outros sintomas, integrando diferentes formas de cuidar, oferecendo suporte para que os pacientes possam viver o mais ativamente possível e ajudando a família e cuidadores no processo de luto ${ }^{3}$. A atenção paliativa envolve tanto a pessoa que está morrendo como aqueles que lhe são próximos, ou seja, familiares e cuidadores ${ }^{14}$.

O desafio provocado pelo luto é vivenciar a morte em vida. Pessoas com estreitos vínculos 
afetivos sentem como se uma parte delas morresse; em alguns casos cria-se um vazio em seu interior que pode ser suportável, porém, irreversível $^{15,16}$

No âmbito da enfermagem cuidar de pacientes fora de possibilidades terapêuticas de cura em processo de luto, pode ser visto de diferentes formas pelos profissionais que trabalham nessa área, uma vez que, cuidar requer mais do que envolvimento real entre o profissional e o paciente, envolve, ainda, a aceitação de nossa própria finitude como ser humano ${ }^{16}$. Contudo, os cuidados paliativos são intrínsecos ao seu exercício diário.

Os cuidados paliativos têm como foco o alívio dos sintomas e a melhoria da qualidade de vida, o que permite um cuidado contínuo, e uma assistência ampla que atenda a pessoa em sua totalidade, considerando-a como um ser biopsicossocial e espiritual. No entanto, para que isso se concretize, torna-se necessário um amparo por parte de uma equipe multidisciplinar com a finalidade de assistir o paciente e família na elaboração do luto ${ }^{17}$. Vale ressaltar que as falas dos participantes da pesquisa traduzem essa percepção quanto à importância desses profissionais nessa área de atuação, visto que, a ação da equipe multidisciplinar deve ocorrer de forma individualizada e focada na dignidade do paciente.

Sob esse prisma a paliação é desenvolvida por uma equipe multiprofissional e visa ofertar um atendimento humanizado e melhor qualidade de vida para pacientes e familiares ${ }^{3}$. A abordagem multidisciplinar denota um aspecto imperativo na filosofia de cuidados paliativos. Esses cuidados direcionam-se para o alívio dos sintomas físicos, psicológicos, espirituais e sociais, o que torna relevante a observação destes problemas sob a ótica de diferentes profissionais para realizar a prestação da assistência. Logo, a prática dos cuidados paliativos tem um caráter multidisciplinar, no sentido de controlar e aliviar, não somente o sofrimento físico, mas o psicossocial e espiritual do paciente, com o objetivo de alcançar um cuidado integral, guiado pelos princípios éticos dos direitos humanos ${ }^{5}$.

É oportuno observar que o suporte da equipe multidisciplinar, dos familiares e da comunidade recebido após a morte de uma pessoa querida é fator extremamente relevante para o enfrentamento do luto. Pesquisas ${ }^{16,17}$ têm mostrado efeitos positivos do suporte social religioso no bem-estar psicológico de enlutados. O templo espiritual, entendido como a comunidade de fiéis que compartilham a mesma fé, proporcionou às pessoas que convivem com esse sentimen- to de perda de um ente querido, espaço de socialização e de expressão de sentimentos, servindo como apoio essencial na elaboração do luto. Um estudo $^{17}$ ressalta, ainda que do ponto de vista prático, que estes resultados devem chamar a atenção para que os profissionais que lidam com situações de luto e morte busquem integrar as crenças religiosas dos enfermos terminais sob seus cuidados na elaboração satisfatória do luto.

Para realizar o trabalho do luto é preciso reconhecer e permitir a expressão de sentimentos presentes. O processo de luto permite elaborar a dor, estimular a comunicação entre pacientes, familiares e profissionais, tendo caráter preventivo. Deve ser direcionado por toda a equipe envolvida na assistência paliativa ao paciente, em especial o enfermeiro. Como membro da equipe de saúde, na atenção paliativa, este exerce papel fundamental, uma vez que acompanha, diariamente, o paciente, desde o momento do diagnóstico, até o final do processo de terminalidade humana, auxiliando-o no enfrentamento de uma realidade impregnada de dor e temor de uma doença terminal.

Categoria III - Comunicação: fonte de dignidade no processo de terminalidade

Nesta categoria, os participantes da pesquisa afirmam que a comunicação é uma ferramenta de grande relevância em cuidados paliativos, por promover uma assistência adequada para que o paciente chegue ao seu destino final com dignidade, conforme se evidencia nos depoimentos:

[...] é uma modalidade de cuidar que valoriza o ser, [...] através da escuta, da comunicação e do respeito ao paciente em sua terminalidade se promove uma assistência qualificada e uma morte digna ao paciente com câncer terminal. (Enf 3 )

É o cuidado prestado ao paciente portador de uma patologia que não tem probabilidade de cura. Esses cuidados vão [...] favorecer uma morte digna ao paciente [...] além de apoiar os familiares $d u$ rante o cuidado [...] Assim, é de fundamental importância facilitar sua prática através de uma comunicação efetiva, [...]. (Enf 4)

Esse cuidado deve promover: Comunicação, [...], diminuir os anseios do paciente. (ENF5)

[...] A equipe deve apoiar, estabelecer vínculos, escutar e promover uma boa comunicação para corresponder os anseios e medos do paciente terminal. (ENF7)

O objetivo maior desse cuidado é uma morte digna onde o paciente seja assistido de forma individualizada e que suas necessidades sejam atendidas [...]. (ENF 8) 
Por meio desses depoimentos revelou-se que a boa comunicação entre o profissional e o paciente poderá promover uma assistência efetiva, facilitar a assistência prestada e minimizar medos e anseios provocados pela doença terminal, o que traz à tona a importância da comunicação na prestação desses cuidados que diminuem os sintomas incapacitantes provocados pela doença.

Portanto, a comunicação é extremamente relevante na relação terapêutica que se estabelece entre a equipe e o paciente, e tem por finalidade proporcionar confiança, para que se possa alcançar uma relação de ajuda efetiva, no qual o paciente e sua família possam expressar temores, angústias, valores e significados ${ }^{3}$.

Nesse sentido, o enfermeiro deve comunicarse efetivamente com o paciente, ouvindo-o sempre que possível, de maneira que possa ajudar a pessoa a revelar os sentimentos que cercam o processo de terminalidade e luto, para que se possa compreender melhor a sua experiência ${ }^{18}$. Tal pensamento corrobora com as falas dos participantes do estudo evidenciando que a comunicação é essencial para estabelecer uma assistência de qualidade promovendo, assim, uma morte digna.

A comunicação entre enfermeiro e paciente tem como objetivo esclarecer dúvidas relacionadas às questões pessoais em situação terminal, permitindo que expresse os aspectos obscuros para ele. Nesse sentido, é importante que o profissional explique sobre procedimentos e condutas a serem seguidas. Por isso, cabe ao enfermeiro valorizar a comunicação com o paciente e, para isso, deve saber como abordar e identificar o que querem expressar com seus gestos, olhares e falas.

Vale salientar que é essencial para o cuidado do paciente que vivencia o processo de finitude que o profissional perceba, compreenda e aplique adequadamente a comunicação verbal e não verbal. A comunicação não verbal é essencial no cuidado ao paciente no processo de terminalidade, pois valoriza a percepção e a compreensão dos sentimentos, desconfianças e aflições, assim como o entendimento e a clareza de gestos, expressões, olhares e linguagem simbólica peculiares de quem está vivenciando seu iminente fim ${ }^{19,20}$.

Estudo realizado com pacientes sob cuidados paliativos evidenciou que a atenção dada aos sinais não verbais pelo profissional para o estabelecimento de um vínculo de confiança afetuosa e valorização da comunicação verbal alegre, que privilegia o otimismo e o bom humor são importantes atributos dos cuidados paliativos. No entanto, percebe-se falta de habilidades e conhecimentos por parte de profissionais de enfer- magem no que diz respeito à comunicação com o paciente fora das possibilidades terapêuticas de cura ${ }^{19}$.

Muitos profissionais desconhecem as técnicas de comunicação terapêutica, e evitam o contato verbal com os pacientes que vivenciam o processo de luto, por não conseguir trabalhar com os seus sentimentos relacionados à situação de morte iminente. Esse aspecto traz à tona, a necessidade de capacitar os profissionais envolvidos na assistência ao paciente terminal, no que tange à comunicação em cuidados paliativos ${ }^{19,20}$.

Para o enfermeiro, não é possível o paciente ter uma morte digna se não existe entre os envolvidos nesse processo demonstração de carinho, respeito e prontidão em estar ao lado desse ser na finitude da vida quando for de sua vontade, de oferecer-lhe uma palavra de conforto, um abraço, um afago ou um ombro para chorar.

\section{Considerações Finais}

Percebe-se que o enfermeiro se refere aos cuidados paliativos com objetividade. Eles reconhecem a importância de um cuidado diferenciado, humanizado, trabalhando de forma multidisciplinar, por meio do qual seja priorizada a qualidade de vida, o conforto, a diminuição da dor, a interação com a família na busca de um cuidado efetivo ao paciente que não responde mais à terapêtica curativa.

O estudo evidenciou que o enfermeiro reconhece que chegar ao destino final com dignidade é um processo que envolve o respeito à condição e às opiniões do paciente como ser que vivencia uma fase da vida com limitações, temores e angústias.

Para a família, a fase terminal de um paciente é um momento de dor e, frequentemente, a aceitação dessa situação é difícil e dolorosa, principalmente, no processo de enlutamento, quando ao perceber que a morte do ente querido não traz somente saudades ou sentimento de incompletude, mas causa também incertezas do futuro. Porquanto, os enfermeiros devem promover os cuidados paliativos a essa família tanto no processo de finitude como no luto.

Os relatos desses cuidadores destacam a comunicação como ferramenta essencial do cuidar. O processo de comunicação se configura como um elemento eficaz do cuidado com o paciente em fase terminal e é de suma importância para a promoção dos cuidados paliativos. Destacam ainda que paliar é proporcionar uma morte digna ao paciente terminal. 
Logo, com base na análise dos depoimentos obtidos, conclui-se que foi possível constatar a importância e a relevância deste estudo para subsidiar futuras investigações e contribuir na formação de conhecimento a fim de ampliar as discussões acerca dos Cuidados Paliativos direcionados ao paciente com câncer em fase terminal.

\section{Colaboradores}

MA Fernandes trabalhou na revisão crítica e na aprovação da versão a ser publicada, ICS Platel, CB Evangelista, MS Lopes e FA Rodrigues trabalharam na análise e interpretação dos dados e na redação do artigo, G Agra trabalhou na revisão crítica e na aprovação da versão a ser publicada.

\section{Referências}

1. Pessini L. Lidando com pedidos de eutanásia: a inserção do filtro paliativo. Rev Bioet 2010; 18(3):54960.

2. Melo AGC, Caponero R. Cuidados paliativos: abordagem contínua e integral. In: Santos FS, organizador. Cuidados paliativos: discutindo a vida, a morte e o morrer. São Paulo: Atheneu; 2009.

3. Melo AGC, Caponero R. O futuro em cuidados paliativos. In: Santos FS. Cuidados paliativos: diretrizes, humanização e alívio de sintomas. São Paulo: Atheneu; 2011.

4. Bertachini L, Pessini L. A importância da dimensão espiritual na prática dos cuidados paliativos. Rev Centro Universitário São Camilo 2010; 4(3):315-323.

5. Silva EP, Sudigursky D. Concepções sobre cuidados paliativos: revisão bibliográfica. Acta paul enferm 2008; 21(3):504-508.

6. Sousa ATO, França JRFS, Costa SFG, Souto CMRM. Cuidados paliativos com pacientes terminais: um enfoque na Bioética. Rev Cuba Enferm 2010; 26(3):123-135.

7. Bardin L. Análise de Conteúdo. Lisboa: Edições 70; 2011.

8. Brasil. Ministério da Saúde(MS). Comissão Nacional de Ética e Pesquisa. Conselho Nacional de Saúde (CNS). Manual Operacional para Comitês de Ética em Pesquisa. Brasília: CNS; 2002. (Série CNS-Cardernos Técnicos, Série A, Normas e Manuais Técnicos, N. 133).

9. Saunders C. Foreword - Oxford textbook of palliative medicine. In: Clark D. Cicely Saunders: selected writings 1958-2004. New York: Oxford University Press; 2006.

10. Silva AF, Issi HB, Motta MGC. A família da criança oncológica em cuidados paliativos: o olhar da equipe de enfermagem. Ciên Cuid Saude 2011; 10(4):820827. 
11. Seki Galheigo SM. O uso da música nos cuidados paliativos: humanizando o cuidado e facilitando o adeus. Interface (Botucatu) 2010; 14(33):273-284.

12. Pessini L.Cuidados Paliativos: Perspectiva Contemporânea. Rev. Bras Cuidados Paliativos 2008; 1(1):2529.

13. Kovács MJ. Desenvolvimento da tanatologia: Estudos sobre a morte e o morrer. Paidéia 2008; 18(41):457-468.

14. Santos DBA, Lattaro RCC, Almeida DA. Cuidados paliativos de enfermagem ao paciente oncológico terminal: revisão da literatura. Rev. Inic Cient Libertas $2011 ; 1(1): 72-84$.

15. Pinheiro TRSP. Avaliação do grau de conhecimento sobre cuidados paliativos e dor dos estudantes de medicina do quinto e sexto anos. Mundo Saúde 2010; 34(3):320-326.

16. Farinasso ALC, Labate RC. Luto, religiosidade e espiritualidade: um estudo clínico-qualitativo com viúvas idosas. Rev. Eletr Enf 2012;14(3):588-595.

17. Rabello CAFG, Rodrigues PHA. Saúde da família e cuidados paliativos infantis: ouvindo os familiares de crianças dependentes de tecnologia. Cien Saude Colet 2010; 15(2):3157-3166.

18. Lopes MEL, Fernandes MA, Platel ICS, Moreira MADM, Duarte MCS, Costa TF. Palliative care: understanding of the assistant nurses. Rev. Enferm UFPE (on line) 2013; 7(1):168-175.

9. Araujo MMT, Silva MJP. Estratégias de comunicação utilizadas por profissionais de saúde na atenção à pacientes sob cuidados paliativos. Rev EsC Enferm USP 2012; 46(3):626-632.

20. Kersun L, Gyi L, Morrison WE. Training in difficult conversations: a national survey of pediatric-oncology and pediatric critical care physicians. J Palliat Med 2009; 12(6):525-530.

Artigo apresentado em 30/04/2013

Aprovado em 22/05/2013

Versão final apresentada em 05/06/2013 